\title{
THE INITIAL VALUE PROBLEM FOR ACOUSTIC GRAVITY WAVES*
}

BY

\author{
GARY MCCARTOR
}

Mission Research Corporation, Santa Barbara, California

\begin{abstract}
The initial value problem for acoustic-gravity waves in atmospheres composed of one or two isothermal layers is solved. For the case of two layers the problem is formulated in an indefinite space.
\end{abstract}

Introduction. Acoustic-gravity waves are small-amplitude disturbances which propagate through a perfect, compressible fluid stratified in a gravitational field. A discussion of the physical properties of these waves including their properties in various parameter regimes, such as the acoustic limit at high frequencies, etc., can be found in reference [1], which also includes an extensive bibliography. In this paper we shall solve the initial value problem for such waves by formulating it as an eigenvalue problem in a Hilbert space, or, in the case of two layers, in an indefinite space. The form of the solution is convenient for application to problems involving a disturbance which is initially strong enough to require nonlinear techniques; if the nonlinear problem can be solved until the disturbance is weak, the end of the nonlinear calculation can serve as an initial condition for the present technique. The method is also of particular value for problems where one is interested in calculating the coupling of an initial configuration to one particular type of mode, such as ducted modes; since these modes appear in our formulation as eigenvectors orthogonal to other modes, the coupling can be calculated without solving the full problem; for example, in the case of an atmosphere composed of two isothermal layers one can calculate the coupling to the ducted modes without solving the full problem. We have found the formulation convenient to use for practical problems and think others may find use for it, but another reason for presenting the results is the possibility that mathematical physicists interested in indefinite spaces may find the space used here to solve a physics problem useful as an example of indefinite spaces.

The case of one isothermal layer.

Hydrodynamic variables and equations. To begin with, we consider the case of one isothermal layer. The hydrostatic solution about which we wish to perturb is given

*Received May 2, 1988.

(C)1989 Brown University 
by

$$
\begin{aligned}
\mathbf{v} & =\mathbf{0}, \\
P & =P_{0} e^{-z / H}, \\
\rho & =\rho_{0} e^{-z / H},
\end{aligned}
$$

where $\mathbf{v}$ is the fluid velocity, $P$ the pressure, and $\rho$ the density. $P_{0}, \rho_{0}$, and $H$ are constants related by $P_{0}=\rho_{0} H g$, where $g$ is the acceleration of gravity. Another quantity we shall use is the sound speed, $c \equiv \sqrt{\gamma g H}$. We now define the variables $\tilde{V}$, $\tilde{P}$, and $\tilde{\rho}$ which will be treated as small, perturbing quantities:

$$
\begin{aligned}
\mathbf{v} & =\tilde{\mathbf{V}} e^{z /(2 H)}, \\
P & =e^{-z / H}\left(P_{0}+\tilde{P} e^{z /(2 H)}\right), \\
\rho & =e^{-z / H}\left(\rho_{0}+\tilde{\rho} e^{z /(2 H)}\right) .
\end{aligned}
$$

We have chosen the exponential factors so that the perturbing functions $\tilde{V}, \tilde{P}, \tilde{\rho}$ will be bounded for all values of $x, z$, and $t$, and, for compact disturbances, will be square integrable functions of $x$ and $z$ for any value of $t$. (This fact is not obvious but is seen in the results below.) For these variables the linearized hydrodynamic equations take on the form:

$$
\begin{aligned}
\frac{\partial \tilde{V}_{z}}{\partial t} & =\frac{1}{2 H \rho_{0}} \tilde{P}-\frac{1}{\rho_{0}} \frac{\partial \tilde{P}}{\partial z}-\frac{g}{\rho_{0}} \tilde{\rho}, \\
\frac{\partial \tilde{P}}{\partial t} & =-\gamma P_{0} \frac{\partial \tilde{V}_{x}}{\partial x}-\gamma P_{0} \frac{\partial \tilde{V}_{z}}{\partial z}-\left(\frac{\gamma}{2}-1\right) \frac{P_{0}}{H} \tilde{V}_{z}, \\
\frac{\partial \tilde{V}_{x}}{\partial t} & =-\frac{1}{\rho_{0}} \frac{\partial \tilde{p}}{\partial x}, \\
\frac{\partial \tilde{\rho}}{\partial t} & =\frac{\rho_{0}}{2 H} \tilde{V}_{z}-\rho_{0} \frac{\partial \tilde{V}_{x}}{\partial x}-\rho_{0} \frac{\partial \tilde{V}_{z}}{\partial z}
\end{aligned}
$$

We assume a time dependence $e^{-i \omega t}$ for each of the variables and obtain

$$
\begin{gathered}
\frac{i}{2 H \rho_{0}} \tilde{P}-\frac{i}{\rho_{0}} \frac{\partial \tilde{P}}{\partial z}-\frac{i g}{\rho_{0}} \tilde{\rho}=\omega \tilde{V}_{z}, \\
-i \gamma P_{0} \frac{\partial \tilde{V}_{x}}{\partial x}-i \gamma P_{0} \frac{\partial \tilde{V}_{z}}{\partial z}-i\left(\frac{\gamma}{2}-1\right) \frac{P_{0}}{H} \tilde{V}_{z}=\omega \tilde{P}, \\
\frac{-i}{\rho_{0}} \frac{\partial \tilde{P}}{\partial x}=\omega \tilde{V}_{x}, \\
\frac{i \rho_{0}}{2 H} \tilde{V}_{z}-i \rho_{0} \frac{\partial \tilde{V}_{x}}{\partial x}-i \rho_{0} \frac{\partial \tilde{V}_{z}}{\partial z}=\omega \tilde{\rho} .
\end{gathered}
$$

We now wish to construct a Hilbert space in which our solution will lie. We arrange the functions into a column vector in the order

$$
|v\rangle=\left(\begin{array}{c}
\tilde{P} \\
\tilde{V}_{z} \\
\tilde{V}_{x} \\
\tilde{\rho}
\end{array}\right)
$$


and define the inner product

$$
\langle a \mid b\rangle=\int_{-\infty}^{\infty} \tilde{P}_{a}^{*} \tilde{P}_{b}+\tilde{V}_{z a}^{*} \tilde{V}_{z b}+\tilde{V}_{x a}^{*} \tilde{V}_{x b}+\tilde{\rho}_{a}^{*} \tilde{\rho}_{b} d x d z
$$

Equation 4 takes on the form

$$
T|v\rangle=\omega|v\rangle
$$

where the operator $T$ is given by

$$
\left(\begin{array}{cccc}
0 & -i \gamma P_{0} \frac{\partial}{\partial z}-i\left(\frac{\gamma}{2}-1\right) \frac{P_{0}}{H} & -i \gamma P_{0} \frac{\partial}{\partial x} & 0 \\
-\frac{i}{\rho_{0}} \frac{\partial}{\partial z}+\frac{i}{2 H \rho_{0}} & 0 & 0 & -\frac{i g}{\rho_{0}} \\
-\frac{i}{\rho_{0}} \frac{\partial}{\partial x} & 0 & 0 & 0 \\
0 & -i \rho_{0} \frac{\partial}{\partial z}+\frac{i \rho_{0}}{2 H} & -i \rho_{0} \frac{\partial}{\partial x} & 0
\end{array}\right) .
$$

This operator is not symmetric; nor does it appear to have any of the other normally desirable properties for operators. We define a new vector space

$$
|a\rangle=\left(\begin{array}{l}
a_{1} \\
a_{2} \\
a_{3} \\
a_{4}
\end{array}\right)
$$

where

$$
\begin{aligned}
& a_{1}=\frac{\tilde{P}}{\sqrt{\gamma} P_{0}}, \\
& a_{2}=\frac{\sqrt{\gamma} \tilde{V}_{z}}{c}, \\
& a_{3}=\frac{\sqrt{\gamma} \tilde{V}_{x}}{c}, \\
& a_{4}=\frac{1}{\sqrt{\gamma(\gamma-1)}}\left(\frac{\tilde{P}}{P_{0}}-\gamma \frac{\tilde{\rho}}{\rho_{0}}\right) .
\end{aligned}
$$

The inner product is

$$
\langle a \mid b\rangle=\int_{-\infty}^{\infty} a_{1}^{*} b_{1}+a_{2}^{*} b_{2}+a_{3}^{*} b_{3}+a_{4}^{*} b_{4} d x d z .
$$

In the new space our eigenvalue problem, Equation (4), becomes

$$
M|a\rangle=\omega|a\rangle
$$

where

where we have defined

$$
M=i c\left(\begin{array}{cccc}
0 & -\frac{\partial}{\partial z}-\Gamma & -\frac{\partial}{\partial x} & 0 \\
-\frac{\partial}{\partial z}+\Gamma & 0 & 0 & \frac{\sqrt{\gamma-1}}{H \gamma} \\
-\frac{\partial}{\partial x} & 0 & 0 & 0 \\
0 & -\frac{\sqrt{\gamma-1}}{H \gamma} & 0 & 0
\end{array}\right)
$$

$$
\Gamma=\frac{1}{H}\left(\frac{1}{2}-\frac{1}{\gamma}\right)
$$

The operator $M$ is self-adjoint. 
Eigenvectors. The spectrum of the operator is all real numbers, $\omega$, which satisfy $\omega^{2}<\omega_{B}^{2}$ or $\omega^{2}>\omega_{A}^{2}$, where

$$
\begin{aligned}
\omega_{A} & =\frac{\gamma g}{2 c}, \\
\omega_{B} & =\sqrt{\gamma-1} \frac{g}{c}
\end{aligned}
$$

$\left(\omega_{A}\right.$ and $\omega_{B}$ are usually called, respectively, the acoustic cutoff frequency and the Brunt-Vaisala frequency). For each point in the spectrum there are an infinite number of degenerate eigenstates which we can label $\left|\omega, k_{x}, j\right\rangle$ where the index $j$ runs from 1 to 4 . These eigenvectors are conveniently given in terms of a vector, $p$, in the finite-dimensional space

$$
\left.\mid p\left(\omega, k_{x}, k_{z}, c\right)\right) \equiv\left(\begin{array}{l}
p_{1} \\
p_{2} \\
p_{3} \\
p_{4}
\end{array}\right)
$$

where

$$
\begin{aligned}
& p_{1} \equiv \frac{\omega\left(1-\left(\frac{\omega_{B}}{\omega}\right)^{2}\right)}{c k_{z}-i \sqrt{\omega_{A}^{2}-\omega_{B}^{2}}}, \\
& p_{2} \equiv 1, \\
& p_{3} \equiv \frac{k_{x} c}{\omega} p_{1}, \\
& p_{4} \equiv-i \frac{\omega_{B}}{\omega},
\end{aligned}
$$

and $k_{z}$ is determined by the dispersion relation

$$
k_{z}^{2}=\frac{1}{c^{2}}\left(\omega^{2}-\omega_{A}^{2}\right)+k_{x}^{2}\left(\left(\frac{\omega_{B}}{\omega}\right)^{2}-1\right) .
$$

The eigenvectors can now be given as

$$
\begin{aligned}
& \left.\left|\omega, k_{x}, 1\right\rangle=\frac{1}{N} \mid p\left(\omega, k_{x}, k_{z}, c\right)\right) e^{i k_{x} x} e^{i k_{z} z}, \\
& \left.\left|\omega, k_{x}, 2\right\rangle=\frac{1}{N} \mid p\left(\omega, k_{x},-k_{z}, c\right)\right) e^{i k_{x} x} e^{-i k_{z} z}, \\
& \left.\left|\omega, k_{x}, 3\right\rangle=\frac{1}{N} \mid p\left(\omega,-k_{x}, k_{z}, c\right)\right) e^{-i k_{x} x} e^{i k_{z} z}, \\
& \left.\left|\omega, k_{x}, 4\right\rangle=\frac{1}{N} \mid p\left(\omega,-k_{x},-k_{z}, c\right)\right) e^{-i k_{x} x} e^{-i k_{z} z} .
\end{aligned}
$$

$N$ is a normalization which we take to be

$$
\begin{aligned}
N^{2} & =(2 \pi)^{2} \frac{1}{\left|\frac{d k_{z}}{d \omega}\right|}(p \mid p) \equiv(2 \pi)^{2} \frac{1}{\left|\frac{d k_{z} \mid}{d \omega}\right|} \sum_{1}^{4}\left|p_{i}\right|^{2} \\
& =8 \pi^{2}\left|\frac{\left(1-\left(\frac{\omega_{B}}{\omega}\right)^{2}\right) \omega k_{z} c^{2}}{c^{2} k_{z}^{2}+\omega_{A}^{2}-\omega_{B}^{2}}\right| .
\end{aligned}
$$

With this choice of $N$ we have

$$
\left\langle\omega^{\prime}, k_{x}^{\prime}, l \mid \omega, k_{x}, m\right\rangle=\delta_{l m} \delta\left(\omega-\omega^{\prime}\right) \delta\left(k_{x}-k_{x}^{\prime}\right) .
$$


For a given value of $\omega$ lying in the spectrum of $M$ we find the range of the parameter $k_{x}$ to be

where

$$
\begin{array}{ll}
k_{x} \geq F(\omega, c), & \omega^{2} \leq \omega_{B}^{2}, \\
k_{x} \leq F(\omega, c), & \omega^{2} \geq \omega_{A}^{2},
\end{array}
$$

$$
F(\omega, c) \equiv\left[\frac{\omega_{A}^{2}-\omega^{2}}{c^{2}\left(\left(\frac{\omega_{B}}{\omega}\right)^{2}-1\right)}\right]^{1 / 2} .
$$

Notice that in the way we have labeled the states both $k_{x}$ and $k_{z}$ are positive numbers.

The solution. The machinery so far accumulated solves the initial value problem for the case of a single isothermal layer. If we know the configuration of the system at time $t=0$ to be $|f(0)\rangle$, the configuration at time $t$ will be $|f(t)\rangle$, given by

$$
|f(t)\rangle=\sum_{j=1}^{4} \iint_{R}\left\langle\omega, k_{x}, j \mid f(0)\right\rangle\left|\omega, k_{x}, j\right\rangle e^{-i \omega t} d k_{x} d \omega,
$$

where the region of integration, $R$, is (see Eq. 22)

$$
R=\left\{\begin{array}{l}
\omega \leq-\omega_{A} ; \quad k_{x} \leq F(\omega, c) \\
-\omega_{B}<\omega<\omega_{B} ; \quad k_{x} \geq F(\omega, c), \\
\omega \geq \omega_{A} ; \quad k_{x} \leq F(\omega, c)
\end{array}\right.
$$

The case of two isothermal layers.

Formulation. We now consider the case where the medium consists of two isothermal layers having two different temperatures (stability requires that the hotter layerthe layer with larger values of $H$ and $c$-be the upper region). We shall use the convention that parameters for the lower region be labeled with the subscript 1 (e.g. $H_{1}$ ) while those for the upper region are labeled with the subscript 2 . We shall assume that the adiabatic constant, $\gamma$, is the same in each layer although this assumption is not necessary. We define the -variables as in Eq. (2) using the value of $H$ and $\rho_{0}$ appropriate to the layer in question $\left(P_{0}\right.$ is the same in the two layers) and then find that Eqs. (3) are satisfied where again we must choose the parameters appropriately. The transformation (10) can then be made for each layer and the dynamical equations can be written as (see Eqs. (12) and (13))

$$
\begin{aligned}
& M\left(c_{1}\right)|a\rangle=\omega|a\rangle, \quad z<0, \\
& M\left(c_{2}\right)|a\rangle=\omega|a\rangle, \quad z>0,
\end{aligned}
$$

where we have taken the discontinuity in temperature to occur at $z=0$.

The (linearized) boundary conditions at the interface between the two layers $(z=$ $0)$ are usually written as

$$
\begin{gathered}
\left.\tilde{V}_{z}\right|_{z=0^{+}}=\left.\tilde{V}_{z}\right|_{z=0^{-}}, \\
\left.\left(\frac{\partial \tilde{P}}{\partial t}-\frac{P_{0}}{H} \tilde{V}_{z}\right)\right|_{z=0^{+}}=\left.\left(\frac{\partial \tilde{P}}{\partial t}-\frac{P_{0}}{H} \tilde{V}_{z}\right)\right|_{z=0^{-}} .
\end{gathered}
$$

These equations result from linearizing the full boundary conditions which require that the $z$-component of the velocity and the pressure be continuous at the (moving) 
interface. For our purposes the appearance of the time derivative is very inconvenient; we use the equations of motion, (3), to eliminate the time derivative and use Eq. (10) to write the result in terms of the $a$-vector. We get

$$
\begin{aligned}
\left.c_{2} a_{2}\right|_{z=0^{+}} & =\left.c_{1} a_{2}\right|_{z=0^{-}}, \\
\left.\left(a_{1}+\frac{1}{\sqrt{\gamma-1}} a_{4}\right)\right|_{z=0^{+}} & =\left.\left(a_{1}+\frac{1}{\sqrt{\gamma-1}} a_{4}\right)\right|_{z=0^{-}} .
\end{aligned}
$$

From the first line of Eq. (27) we see that the function $a_{2}$ must be discontinuous across the interface; from the second line we see that $a_{1}$ and $a_{4}$ may be separately discontinuous so long as the particular linear combination of them is continuous (it is this last possibility that makes the trouble). The difficulty which prevents the extension of our one-layer methodology to two layers from being completely straightforward is that the boundary conditions (27) make the operator $M$ nonsymmetric in the space with the inner product $\langle\cdot \mid \cdot\rangle$ defined by Eq. (11). The operator $i \frac{\partial}{\partial z}$ is usually not symmetric if there are boundaries across which functions change discontinuously. The nonsymmetry of $M$ in the inner product $\langle\cdot \mid \cdot\rangle$ is shown explicitly in the appendix.

A means of alleviating the problem is to redefine the inner product. Given two vectors in $a$-space we define

$$
\{a \mid b\} \equiv \int_{-\infty}^{\infty}\left(\int_{-\infty}^{\infty}\left(a_{1}^{*} b_{1}+a_{2}^{*} b_{2}+a_{3}^{*} b_{3}+a_{4}^{*} b_{4}\right) d z-\left.\frac{\gamma}{\gamma-1}\left[a_{4}^{*} H b_{4}\right]\right|_{z=0^{-}} ^{z=0^{+}}\right) d x .
$$

The form (28) is linear in the right-hand element and antilinear in the left-hand element but it is not positive definite when $a$ and $b$ are the same so we have an indefinite space. In the appendix we show that the operator $M$ is symmetric in the new space.

Eigenvectors. The eigenmode structure of the two-layer case is substantially more complicated than that for the one-layer case. Figure 1 is useful in sorting out the structure. The particular case shown there is $c_{1}=0.5 ; c_{2}=1 ; \omega_{A_{1}}=4 / \sqrt{3} ; \omega_{B_{1}}=2$; $\omega_{A_{2}}=2 / \sqrt{3} ; \omega_{B_{2}}=1$ (these numbers imply that $\gamma=4 / 3$ ). The qualitative aspects of the eigenmode structure are the same as long as $c_{1}<c_{2}$ (which is necessary for stability) and $1<\gamma<2$. The solid curve is the line

$$
k_{x}=\frac{\omega}{c_{1}}\left[\frac{\omega_{A_{1}}^{2}-\omega^{2}}{\omega_{B_{1}}^{2}-\omega^{2}}\right]^{1 / 2} .
$$

For values of $k_{x}$ and $\omega$ which lie on this line, $k_{z_{1}}=0$. If $\omega<\omega_{B_{1}}$ then we find that if $k_{x}$ is above the line, $k_{z_{1}}^{2}$ is positive; if $k_{x}$ is below the line, $k_{z_{1}}^{2}$ is negative. If $\omega>\omega_{A_{1}}$ then if $k_{x}$ is below the line, $k_{z_{1}}^{2}$ is positive while if $k_{x}$ is above the line, $k_{z_{1}}^{2}$ is negative. If $\omega_{B_{1}}<\omega<\omega_{A_{1}}, k_{z_{1}}^{2}$ is negative for all values of $k_{x}$. Similar remarks apply for $k_{z_{2}}^{2}$ where the relevant line is shown by the dashed curve in Fig. 1; the equation for the line is

$$
k_{x}=\frac{\omega}{c_{2}}\left[\frac{\omega_{A_{2}}^{2}-\omega^{2}}{\omega_{B_{2}}^{2}-\omega^{2}}\right]^{1 / 2} .
$$




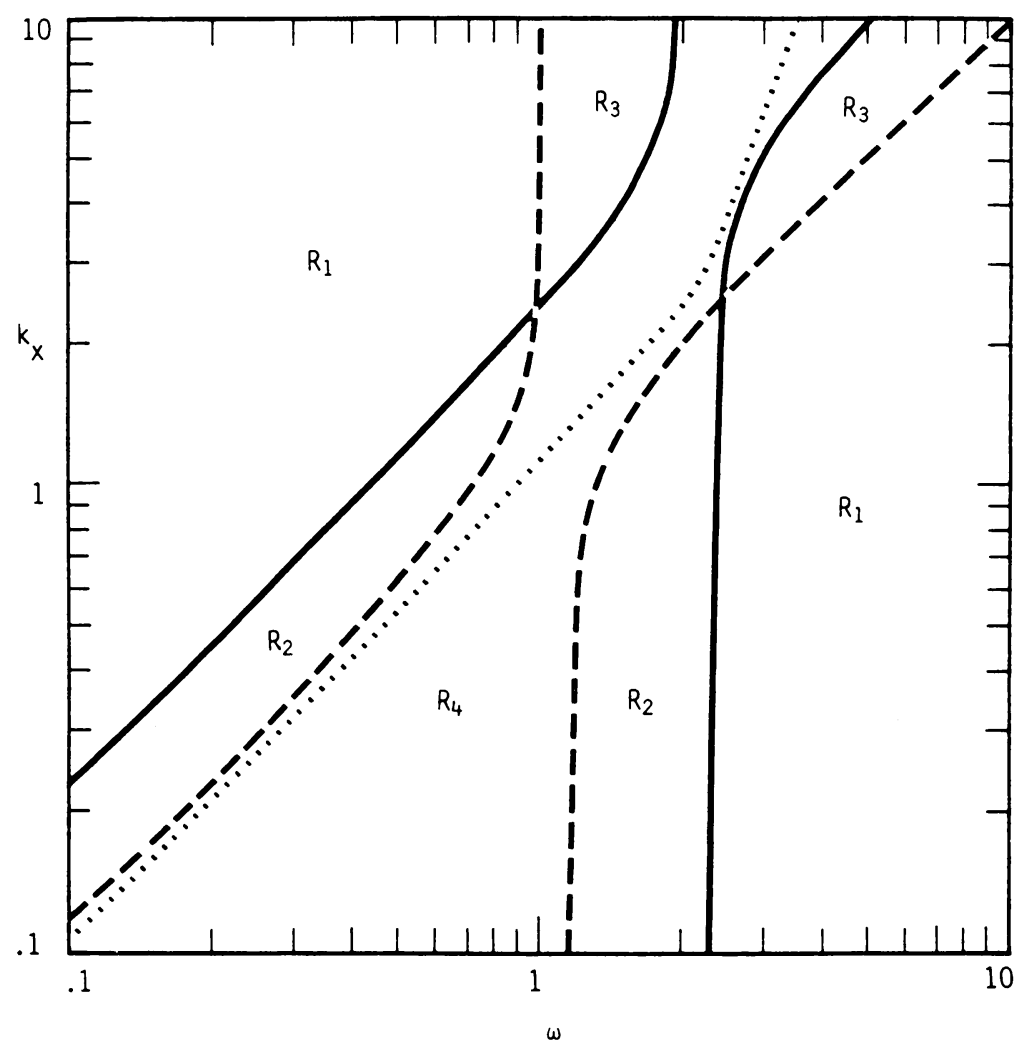

Fig. 1. The mode structure of the two-layer case

The two curves cross at two values of $\omega$ given by

$$
\omega_{c^{ \pm}}^{2}=\frac{1}{2} \frac{\omega_{A_{1}}^{2}\left(c_{1}^{2}+c_{2}^{2}\right)}{c_{2}^{2}}\left\{1 \pm\left[1-\frac{16(\gamma-1)}{\gamma^{2}} \frac{c_{1}^{2} c_{2}^{2}}{\left(c_{1}^{2}+c_{2}^{2}\right)^{2}}\right]^{1 / 2}\right\} .
$$

Let us call the solution to Eq. (29) $k_{x_{1}}$ and the solution to (30) $k_{x_{2}}$. (Note that these do not indicate the value of $k_{x}$ in regions 1 and $2 ; k_{x}$ must have the same value in each region in order to satisfy the boundary conditions.) First consider the region $R_{1}$ which consists of

$$
R_{1} \in \begin{cases}\omega<\omega_{c^{-}} & : k_{x}>k_{x_{1}}, \\ \omega_{c^{-}}<\omega<\omega_{B_{2}} & : k_{x}>k_{x_{2}}, \\ \omega_{A_{1}}<\omega<\omega_{c^{+}} & : k_{x}<k_{x_{1}}, \\ \omega_{c^{+}}<\omega & : k_{x}<k_{x_{2}} .\end{cases}
$$

Also included in $R_{1}$ are all points in the image region generated by the substitution $\omega \rightarrow-\omega$. For each point in $R_{1}$ there are four eigenvectors, called the freely propagating modes. These modes exhibit oscillatory behavior in both regions 1 and 2 and are much like the modes for the one-layer case. As shown in the figure the region $R_{2}$ 
consists of:

$$
R_{2} \in \begin{cases}\omega<\omega_{c^{-}} & : k_{x_{2}}<k_{x}<k_{x_{1}} \\ \omega_{A_{2}}<\omega<\omega_{A_{1}} & : k_{x}<k_{x_{2}} \\ \omega_{A_{1}}<\omega<\omega_{c^{+}} & : k_{x_{1}}<k_{x}<k_{x_{2}}\end{cases}
$$

$R_{2}$ also includes the image region created by the substitution $\omega \rightarrow-\omega$. At each point in $R_{2}$ there are two eigenvectors, called mixed modes. These modes exhibit oscillatory behavior in the $z$-variable in layer 2 but exponentially decaying behavior in $z$ in layer 1 . Region $R_{3}$ includes

$$
R_{3} \in \begin{cases}\omega_{c-}<\omega<\omega_{B_{2}} & : k_{x_{1}}<k_{x}<k_{x_{2}} \\ \omega_{B_{2}}<\omega<\omega_{B_{1}} & : k_{x_{1}}<k_{x} \\ \omega_{c^{+}}<\omega & : k_{x_{2}}<k_{x}<k_{x_{1}}\end{cases}
$$

plus the image under $\omega \rightarrow-\omega$. At each point in $R_{3}$ there are two eigenvectors also called mixed modes. These modes exhibit oscillatory behavior in $z$ in layer 1 but exponentially decaying behavior in $z$ in layer 2. The points in the half-plane $k_{x}>0$ which are not in $R_{1}, R_{2}$, or $R_{3}$ are in $R_{4}$. There are no eigenstates for points in $R_{4}$ except for the dotted line shown in the figure which corresponds to the solution to the equation

$$
\frac{k_{x}^{2} c_{1}^{2}-\omega^{2}}{k_{x}^{2} c_{2}^{2}-\omega^{2}}-\frac{\frac{2}{\gamma} \frac{k_{x}^{2} c_{1} \omega_{i_{1}}}{\omega^{2}}-\frac{\omega_{1_{1}}}{c_{1}}+i k_{z_{1}}}{\frac{2}{\gamma} \frac{k_{x}^{2} c_{2} \omega_{1_{2}}}{\omega^{2}}-\frac{\omega_{1_{2}}}{c_{2}}-i k_{z_{2}}}=0
$$

$k_{z_{1}}$ and $k_{z_{2}}$ are solutions to the dispersion relation, (17), in their respective layers (recall that since we are in the region $R_{4}, k_{z_{1}}$ and $k_{z_{2}}$ are both pure imaginary). At each point on the line, (35), there are two eigenvectors. These are called the ducted modes [2]; they are exponentially decaying in both layers 1 and 2.

We shall label the eigenvectors in $R_{1},\left|\omega, k_{x}, j\right\rangle_{F}$ where $j$ runs from 1 to 4 . A convenient choice is

$$
\left|\omega, k_{x}, 1\right\rangle_{F} \sim\left\{\begin{array}{l}
\left.\mid p\left(\omega, k_{x},-k_{z_{1}}, c_{1}\right)\right) e^{i k_{x} x} e^{-i k_{z_{1}} z}, \quad z<0 \\
\left.A \mid p\left(\omega, k_{x}, k_{z_{2}}, c_{2}\right)\right) e^{i k_{x} x} e^{i k_{z_{2}} z} \\
\left.+B \mid p\left(\omega, k_{x},-k_{z_{2}}, c_{2}\right)\right) e^{i k_{x} x} e^{-i k_{z_{2} z} z}, \quad z>0
\end{array}\right.
$$

$\left|\omega, k_{x}, 2\right\rangle_{F}$ is proportional to the RHS of (36) with $k_{x}$ everywhere replaced by $-k_{x}$. For the other two states we have

$$
\left|\omega, k_{x}, 3\right\rangle_{F} \sim\left\{\begin{array}{l}
\left.\left.C \mid p\left(\omega, k_{x}, k_{z_{1}}, c_{1}\right)\right) e^{i k_{1} x} e^{i k_{z_{1}} z}+D \mid p\left(\omega, k_{x},-k_{z_{1}}, c_{1}\right)\right) e^{i k_{1} x} e^{-i k_{z_{1}} z}, \\
\left.\mid p\left(\omega, k_{x}, k_{z_{2}}, c_{2}\right)\right) e^{i k_{x} x} e^{i k_{z_{2}} z}, \quad z>0 .
\end{array}\right.
$$


$\left|\omega, k_{x}, 4\right\rangle_{F}$ is proportional to the RHS of (37) with $k_{x}$ everywhere replaced with $-k_{x}$. The coefficients $A, B, C$, and $D$ are determined by the boundary conditions. We get

$$
\begin{aligned}
& A=\frac{p_{1}\left(\omega, k_{x},-k_{z_{1}}, c_{1}\right)-\frac{c_{1}}{c_{2}} p_{1}\left(\omega, k_{x},-k_{z_{2}}, c_{2}\right)+\frac{1}{\sqrt{\gamma-1}} \frac{i \omega_{B_{1}}}{\omega}\left(\frac{c_{1}^{2}}{c_{2}^{2}}-1\right)}{p_{1}\left(\omega, k_{x_{1}}, k_{z_{2}}, c_{2}\right)-p_{1}\left(\omega, k_{x},-k_{z_{2}}, c_{2}\right)}, \\
& B=\frac{c_{1}}{c_{2}}-A, \\
& C=\frac{p_{1}\left(\omega, k_{x}, k_{z_{2}}, c_{2}\right)-\frac{c_{2}}{c_{1}} p_{1}\left(\omega, k_{x},-k_{z_{1}}, c_{1}\right)+\frac{1}{\sqrt{\gamma-1}} \frac{i \omega_{B_{2}}}{\omega}\left(\frac{c_{2}^{2}}{c_{1}^{2}}-1\right)}{p_{1}\left(\omega, k_{x_{1}}, k_{z_{1}}, c_{1}\right)-p_{1}\left(\omega, k_{x},-k_{z_{1}}, c_{1}\right)}, \\
& D=\frac{c_{2}}{c_{1}}-C .
\end{aligned}
$$

These eigenvectors have two convenient properties: they are mutually orthogonal; subsets of them analytically continue into the mixed mode eigenvectors of regions $R_{2}$ and $R_{3}$. In particular, when we cross from region $R_{1}$ to region $R_{2}$, we label the eigenvectors $\left|\omega, k_{x}, j\right\rangle_{M_{2}}$ where $j$ is 1 or 2 and find that these are proportional, respectively, to the analytic continuation of $\left|\omega, k_{x}, 1\right\rangle_{F}$ and $\left|\omega, k_{x}, 2\right\rangle_{F}$. If we cross from region $R_{1}$ to region $R_{3}$ we label the states $\left|\omega, k_{x}, j\right\rangle_{M_{3}}$ where $j$ is 1 or 2 and find that they are proportional, respectively, to the analytic continuation of $\left|\omega, k_{x}, 3\right\rangle_{F}$ and $\left|\omega, k_{x}, 4\right\rangle_{F}$. The normalization of the states does not analytically continue across the boundaries. We define normalization functions:

$$
\begin{aligned}
& N_{F}^{2} \equiv(2 \pi)^{2}\left(\left|\frac{\left(1-\left(\frac{\omega_{B_{1}}}{\omega}\right)^{2}\right) \omega k_{z_{1}} c_{1}}{c_{1}^{2} k_{z_{1}}^{2}+\omega_{A_{1}}^{2}-\omega_{B_{1}}^{2}}\right|+\left|\frac{\left(1-\left(\frac{\omega_{B_{2}}}{\omega}\right)^{2}\right) \omega k_{z_{2}} c_{2}}{c_{2}^{2} k_{z_{2}}^{2}+\omega_{A_{2}}^{2}-\omega_{B_{2}}^{2}}\right|\left(|A|^{2}+|B|^{2}\right)\right), \\
& j=1,2, \\
& N_{F}^{2} \equiv(2 \pi)^{2}\left(\left|\frac{\left(1-\left(\frac{\omega_{B_{2}}}{\omega}\right)^{2}\right) \omega k_{z_{2}} c_{2}}{c_{2}^{2} k_{z_{2}}^{2}+\omega_{A_{2}}^{2}-\omega_{B_{2}}^{2}}\right|+\left|\frac{\left(1-\left(\frac{\omega_{B_{1}}}{\omega}\right)^{2}\right) \omega k_{z_{1}} c_{1}}{c_{1}^{2} k_{z_{1}}^{2}+\omega_{A_{1}}^{2}-\omega_{B_{1}}^{2}}\right|\left(|C|^{2}+|D|^{2}\right)\right), \\
& N_{M_{2}}^{2} \equiv(2 \pi)^{2}\left|\frac{\left(1-\left(\frac{\omega_{B_{2}}}{\omega}\right)^{2}\right) \omega k_{z_{2}} c_{2}}{c_{2}^{2} k_{z_{2}}^{2}+\omega_{A_{2}}^{2}-\omega_{B_{2}}^{2}}\right|\left(|A|^{2}+|B|^{2}\right), \\
& N_{M_{3}}^{2} \equiv(2 \pi)^{2}\left|\frac{\left(1-\left(\frac{\omega_{B_{1}}}{\omega}\right)^{2}\right) \omega k_{z_{1}} c_{1}}{c_{1}^{2} k_{z_{1}}^{2}+\omega_{A_{1}}^{2}-\omega_{B_{2}}^{2}}\right|\left(|C|^{2}+|D|^{2}\right) .
\end{aligned}
$$

The vectors $\left|\omega, k_{x}, j\right\rangle_{F},\left|\omega, k_{x}, j\right\rangle_{M_{2}}$, or $\left|\omega, k_{x}, j\right\rangle_{M_{3}}$ are given by the RHS of (36) divided by the appropriate normalization factor from (39); with this normalization we have

$$
\left\{\omega^{\prime}, k_{x}^{\prime}, l \mid \omega, k_{x}, m\right\}=\delta_{l m} \delta\left(k_{x}-k_{x}^{\prime}\right) \delta\left(\omega-\omega^{\prime}\right)
$$


for all the vectors whether of type $F, M_{2}$, or $M_{3}$.

For each point on the dotted line in region $R_{4}$ there are two eigenvectors corresponding to ducted modes traveling in the plus or minus $x$-directions. Since there is only one value of $k_{x}$ for a given $\omega$ we could label the eigenvectors with $\omega$; it is somewhat more convenient to label them with $k_{x}$ thus obtaining four eigenvectors for each $k_{x}$-two for the two $x$-directions times two for the two possible signs for $\omega$ each of which corresponds to the given value for $k_{x}$. We shall call these vectors $\left|k_{x}, j\right\rangle_{D}$ where $j$ runs from 1 to 4 and

$$
\left|k_{x}, 1\right\rangle_{D}= \begin{cases}\left.\frac{1}{c_{1} N_{D}} \mid p\left(|\omega|, k_{x},-k_{z_{1}}, c_{1}\right)\right) e^{i k_{x} x} e^{-i k_{z_{1}} z}, & z<0, \\ \left.\frac{1}{c_{2} N_{D}} \mid p\left(|\omega|, k_{x}, k_{z_{2}}, c_{2}\right)\right) e^{i k_{x} x} e^{i k_{z} z}, & z>0 .\end{cases}
$$

$\left|k_{x}, 2\right\rangle_{D}$ is the same function with the replacement $k_{x} \rightarrow-k_{x}$ on the RHS.

$$
\left|k_{x}, 3\right\rangle_{D}=\left\{\begin{array}{l}
\left.\frac{1}{c_{1} N_{D}} \mid p\left(-|\omega|, k_{x},-k_{z_{1}}, c_{1}\right)\right) e^{i k_{x} x} e^{-i k_{z_{1}} z}, \\
\left.\frac{1}{c_{2} N_{D}} \mid p\left(-|\omega|, k_{x}, k_{z_{2}}, c_{2}\right)\right) e^{i k_{x} x} e^{i k_{z} z} .
\end{array}\right.
$$

$\left|k_{x}, 4\right\rangle_{D}$ is the same function with the replacement $k_{x} \rightarrow-k_{x}$ on the RHS. We choose the normalization to be

$$
\begin{aligned}
& \left.N_{D}^{2}=\left.2 \pi\left\{\| p\left(|\omega|, k_{x},-k_{z_{1}}, c_{1}\right)\right)\right|^{2} \frac{1}{2 c_{1}\left|k_{z_{1}}\right|}+\| p\left(|\omega|, k_{x}, k_{z_{2}}, c_{2}\right)\right)\left.\right|^{2} \frac{1}{2 c_{2}\left|k_{z_{2}}\right|} \\
& +\frac{\gamma}{\gamma-1}\left(H_{1}\left|p_{4}\left(|\omega|, k_{x},-k_{z_{1}}, c_{1}\right)\right|^{2}-H_{2}\left|p_{4}\left(|\omega|, k_{x}, k_{z_{2}}, c_{2}\right)\right|^{2}\right\}
\end{aligned}
$$

for $j=1,2$ and similarly for $j=3,4$ with $|\omega| \rightarrow-|\omega|$. With this choice we have

$$
{ }_{D}\left\{k_{x}^{\prime}, l \mid k_{x}, m\right\}_{D}=\delta_{l m} \delta\left(k_{x}-k_{x}^{\prime}\right) .
$$

The solution. If, at time $t=0$, the configuration of the system is $|f(0)\rangle$, at time $t$ it will be $|f(t)\rangle$ where

$$
\begin{aligned}
|f(t)\rangle= & \sum_{j=1}^{4} \int_{R_{1}} \int_{F}\left\{\omega, k_{x}, j \mid f(0)\right\}\left|\omega, k_{x}, j\right\rangle_{F} e^{-i \omega t} d k_{x} d \omega \\
& +\sum_{j=1}^{2} \int_{R_{2}} \int_{M_{2}}\left\{\omega, k_{x}, j \mid f(0)\right\}\left|\omega, k_{x}, j\right\rangle_{M_{2}} e^{-i \omega t} d k_{x} d \omega \\
& +\sum_{j=1}^{2} \int_{R_{3}} \int_{M_{3}}\left\{\omega, k_{x}, j \mid f(0)\right\}\left|\omega, k_{x}, j\right\rangle_{M_{3}} e^{-i \omega t} d k_{x} d \omega \\
& +\sum_{j=1}^{2} \int_{0}^{\infty}{ }_{D}\left\{k_{x}, j \mid f(0)\right\}\left|k_{x}, j\right\rangle_{D} e^{-i \omega\left(k_{x}\right) t} d k_{x} \\
& +\sum_{j=3}^{4} \int_{0}^{\infty}\left\{k_{x}, j \mid f(0)\right\}\left|k_{x}, j\right\rangle_{D} e^{i \omega\left(k_{x}\right) t} d k_{x} .
\end{aligned}
$$

In the last two lines the function $\omega\left(k_{x}\right)$ is determined by Eq. (35).

Remarks. It may happen that the dotted line in the figure (the solution to Eq. (35)) crosses one of the lines which defines the boundary of $R_{4}$. If that happens it will cross in two places and there is a gap in the last two integrations in Eq. (44). If 
one wishes to solve problems with radial symmetry the necessary replacement is

$$
\begin{gathered}
\left(\begin{array}{l}
P_{1} \\
P_{2} \\
P_{3} \\
P_{4}
\end{array}\right) e^{ \pm i k_{x} x} \rightarrow\left(\begin{array}{l}
P_{1} J_{0}\left(k_{r} r\right) \\
P_{2} J_{0}\left(k_{r} r\right) \\
P_{3} J_{1}\left(k_{r} r\right) \\
P_{4} J_{0}\left(k_{r} r\right)
\end{array}\right), \\
d x d z \rightarrow k_{r} r d r d z,
\end{gathered}
$$

where $k_{r}$ satisfies all the equations $k_{x}$ satisfies in the Cartesian case. Note that all the eigenvectors of $M$ have positive norm even though the space is an indefinite space, a result which might seem impossible at first thought.

Acknowledgment. The author wishes to acknowledge useful discussions with Dr. W. R. Wortman and support from the Defense Advanced Research Projects Agency under DARPA Order 3841 and from the Defense Nuclear Agency under contract DNA001-84-C-0253.

Appendix. In this appendix we will discuss in detail the considerations which lead us to use the inner product defined in Eq. 28. The operator $M$ was defined to be

$$
M=i c\left(\begin{array}{cccc}
0 & -\frac{\partial}{\partial z}-\frac{1}{2 H}+\frac{1}{\gamma H} & -\frac{\partial}{\partial x} & 0 \\
-\frac{\partial}{\partial z}+\frac{1}{2 H}-\frac{1}{\gamma H} & 0 & 0 & \frac{\sqrt{\gamma-1}}{\gamma H} \\
-\frac{\partial}{\partial x} & 0 & 0 & 0 \\
0 & -\frac{\sqrt{\gamma-1}}{H \gamma} & 0 & 0
\end{array}\right)
$$

where $H$ and $c$ take on the values $H_{1}$ and $c_{1}$ if $z<0$ and the values $H_{2}$ and $c_{2}$ if $z>0$. The inner product for the one-layered case was taken to be

$$
\langle a \mid b\rangle=\int_{-\infty}^{\infty} \int_{-\infty}^{\infty}\left(a_{1}^{*} b_{1}+a_{2}^{*} b_{2}+a_{3}^{*} b_{3}+a_{4}^{*} b_{4}\right) d x d z .
$$

We shall first show that the operator $M$ is not symmetric in the space whose inner product is defined by Equation A-2. We have

$$
\begin{array}{r}
\langle a \mid M b\rangle=\int_{-\infty}^{\infty} i\left[c _ { 1 } \int _ { - \infty } ^ { 0 } \left(a_{1}^{*}\left(-\frac{\partial b_{2}}{\partial z}-\frac{\gamma-2}{2 \gamma H_{1}} b_{2}-\frac{\partial b_{3}}{\partial x}\right)\right.\right. \\
+a_{2}^{*}\left(-\frac{\partial b_{1}}{\partial z}-\frac{2-\gamma}{2 \gamma H_{1}} b_{1}+\frac{\sqrt{\gamma-1}}{H_{1} \gamma} b_{4}\right) \\
\left.+a_{3}^{*}\left(-\frac{\partial b_{1}}{\partial x}\right)+a_{4}^{*}\left(-\frac{\sqrt{\gamma-1}}{\gamma H_{1}} b_{2}\right)\right) d z \\
+c_{2} \int_{0}^{\infty}\left(a_{1}^{*}\left(-\frac{\partial b_{2}}{\partial z}-\frac{\gamma-2}{2 \gamma H_{2}} b_{2}-\frac{\partial b_{3}}{\partial x}\right)\right. \\
\quad+a_{2}^{*}\left(-\frac{\partial b_{1}}{\partial z}-\frac{2-\gamma}{2 \gamma H_{2}} b_{1}+\frac{\sqrt{\gamma-1}}{\gamma H_{2}} b_{4}\right) \\
\left.\left.\quad+a_{3}^{*}\left(-\frac{\partial b_{1}}{\partial x}\right)+a_{4}^{*}\left(-\frac{\sqrt{\gamma-1}}{\gamma H_{2}} b_{2}\right)\right) d z\right] d x .
\end{array}
$$


For the conjugate matrix element we have

$$
\begin{aligned}
\langle M a \mid b\rangle=\int_{-\infty}^{\infty}-i\left[c _ { 1 } \int _ { - \infty } ^ { 0 } \left(b_{1}\right.\right. & \left(-\frac{\partial a_{2}^{*}}{\partial z}-\frac{\gamma-2}{2 \gamma H_{1}} a_{2}^{*}-\frac{\partial a_{3}^{*}}{\partial x}\right) \\
+ & b_{2}\left(-\frac{\partial a_{1}^{*}}{\partial z}-\frac{2-\gamma}{2 \gamma H_{1}} a_{1}^{*}+\frac{\sqrt{\gamma-1}}{H_{1}} a_{4}^{*}\right) \\
& \left.+b_{3}\left(-\frac{\partial a_{1}^{*}}{\partial x}\right)+b_{4}\left(-\frac{\sqrt{\gamma-1}}{\gamma H_{1}} a_{2}^{*}\right)\right) d z \\
+c_{2} \int_{0}^{\infty}\left(b_{1}\left(-\frac{\partial a_{2}^{*}}{\partial z}-\frac{\gamma-2}{2 \gamma H_{2}} a_{2}^{*}-\frac{\partial a_{3}^{*}}{\partial x}\right)\right. & +b_{2}\left(-\frac{\partial a_{1}^{*}}{\partial z}-\frac{2-\gamma}{2 \gamma H_{2}} a_{1}^{*}+\frac{\sqrt{\gamma-1}}{\gamma H_{2}} a_{4}^{*}\right) \\
& \left.\left.+b_{3}\left(-\frac{\partial a_{1}^{*}}{\partial x}\right)+b_{4}\left(-\frac{\sqrt{\gamma-1}}{\gamma H_{2}} a_{2}^{*}\right)\right) d z\right] d x
\end{aligned}
$$

We now calculate (integrating the $\partial / \partial x$ terms in $\langle a \mid M b\rangle$ by parts):

$$
\begin{aligned}
\langle a \mid M b\rangle-\langle M a \mid b\rangle= & \int_{-\infty}^{\infty} i\left[c_{1} \int_{-\infty}^{0}\left(-a_{1}^{*} \frac{\partial b_{2}}{\partial z}-a_{2}^{*} \frac{\partial b_{1}}{\partial z}-b_{1} \frac{\partial a_{2}^{*}}{\partial z}-b_{2} \frac{\partial a_{1}^{*}}{\partial z}\right) d z\right. \\
& \left.+c_{2} \int_{0}^{\infty}\left(-a_{1}^{*} \frac{\partial b_{2}}{\partial z}-a_{2}^{*} \frac{\partial b_{1}}{\partial z}-b_{1} \frac{\partial a_{2}^{*}}{\partial z}-b_{2} \frac{\partial a_{1}^{*}}{\partial z}\right) d z\right] d x
\end{aligned}
$$

Integrating the second two terms in each pair of brackets by parts we have

$$
\langle a \mid M b\rangle-\langle M a \mid b\rangle=-\int_{-\infty}^{\infty}\left(\left.i c_{1}\left[a_{1}^{*} b_{2}+a_{2}^{*} b_{1}\right]\right|_{0^{-}}-\left.i c_{2}\left[a_{1}^{*} b_{2}+a_{2}^{*} b_{1}\right]\right|_{0^{+}}\right) d x .
$$

Looking at the boundary conditions given in Eq. 27 in the text we see that there are vectors for which

$$
\langle a \mid M b\rangle-\langle M a \mid b\rangle \neq 0
$$

in the inner product defined by Eq. A-2.

The solution to the problem of the nonsymmetry of the operator $M$ in the standard $L_{2}$ space is to subtract off the remainder term in Eq. A-6 within the inner product. That this is possible is due essentially to the fact that although the boundary condition as written in Eq. 26 involves the time derivative, we can rewrite it in such a way that it does not, as is done in Eq. 27. We now redefine the inner product as (see Eq. 28)

$$
\begin{aligned}
\{a \mid b\}= & \int_{-\infty}^{\infty}\left(\int_{-\infty}^{\infty}\left(a_{1}^{*} b_{1}+a_{2}^{*} b_{2}+a_{3}^{*} b_{3}+a_{4}^{*} b_{4}\right) d z\right. \\
& -\frac{\gamma}{\gamma-1}\left(\left.a_{4}^{*} H_{2} b_{4}\right|_{0^{+}}-\left.a_{4}^{*} H_{1} b_{4}\right|_{0^{-}}\right) d x .
\end{aligned}
$$


Repeating the arguments which lead to Eq. A-6 but using the new definition of the inner product we find

$$
\begin{aligned}
& \{a \mid M b\}-\{M a \mid b\} \\
& =-\int_{-\infty}^{\infty} i\left(\left.\left[a_{1}^{*} b_{2} c\right]\right|_{0^{+}} ^{0^{-}}+\left.\left[a_{2}^{*} b_{1} c\right]\right|_{0^{+}} ^{0^{-}}\right. \\
& \quad-\frac{\gamma}{\gamma-1}\left(\left.a_{4}^{*} i c_{2} \frac{\sqrt{\gamma-1}}{\gamma} b_{2}\right|_{0^{+}}-\left.a_{4}^{*} i c_{1} \frac{\sqrt{\gamma-1}}{\gamma} b_{2}\right|_{0^{-}}\right. \\
& \left.\left.\quad+\left.b_{4} i c_{2} \frac{\sqrt{\gamma-1}}{\gamma} a_{2}^{*}\right|_{0^{+}}-\left.b_{4} i c_{1} \frac{\sqrt{\gamma-1}}{\gamma} a_{2}^{*}\right|_{0^{-}}\right)\right) d x .
\end{aligned}
$$

The boundary conditions (27) now give

$$
\{a \mid M b\}-\{M a \mid b\}=0
$$

so that the operator $M$ is symmetric with the form (A-7) taken as an inner product. The form, (A-7), has most of the properties of an inner product: it is linear in the right-hand element; it is antilinear in the left-hand element; it is real when the elements are the same, but, it is not positive definite when the two elements are the same. The space we are working in is thus an indefinite space.

\section{REFERENCES}

[1] The literature on acoustic-gravity waves is extensive. See S. H. Francis, J. Atmos. and Terr. Phys. 37, 1011, 1975

[2] G. D. Thome, J. Geophys. Res. 73, 6319, 1967 